\title{
Polarization division multiplexing system quality in the presence of polarization effects
}

\section{Krzysztof Perlicki}

Received: 6 January 2010 / Accepted: 3 September 2010 / Published online: 22 September 2010

(C) The Author(s) 2010. This article is published with open access at Springerlink.com

\begin{abstract}
In this paper the Polarization Division Multiplexing system testing method is presented. Presented method is based on simultaneous Polarization Mode Dispersion and Polarization Dependent Loss effects generation. These effects are generated by means of only one device that simulates the statistical nature of polarization effects of a real optical fiber link. The Polarization Division Multiplexing system is investigated by means of Polarization Mode Dispersion/Polarization Dependent Loss emulator.
\end{abstract}

Keywords Birefringence · Polarization - Optical fiber · Polarization Mode Dispersion · Polarization Dependent Loss · Polarization Division Multiplexing

\section{Introduction}

Polarization effects due to interaction between Polarization Mode Dispersion (PMD) and Polarization Dependent Loss (PDL) can significantly impair optical fiber transmission systems. When PMD and PDL are both present, they interact and should be studied together (Palmer 2006). Emulating of statistical nature of PMD and PDL is one way to test and verify new high bit rates transmission systems in the presence of PMD and PDL effects. Because of this the statistical emulator (than deterministic one) is more adequate for Polarization Division Multiplexing (PDM) transmission testing. Statistical polarization effect emulators should accurately reproduce the statistics of the polarization effects that a signal would see on a real link, as well as have good stability and repeatability (Palmer 2006; Lima 2001). The low

\footnotetext{
K. Perlicki $(\bowtie)$

Institute of Telecommunications, Warsaw University of Technology,

Nowowiejska 15/19, 00-665 Warsaw, Poland

e-mail: perlicki@tele.pw.edu.pl

K. Perlicki

Research and Development Centre Branch Telekomunikacja Polska, Obrzezna 7, 02-691 Warsaw, Poland

e-mail: Krzysztof.Perlicki@telekomunikacja.pl
} 
Table 1 DGD and PDL values for each birefringence segment

\begin{tabular}{lcc}
\hline Birefringence segment & DGD [ps] & PDL [dB] \\
\hline 1 & 13.01 & 0.233 \\
2 & 11.11 & 0.226 \\
3 & 11.49 & 0.317 \\
4 & 7.66 & 0.188 \\
5 & 10.66 & 0.226 \\
6 & 11.16 & 0.227 \\
7 & 14.58 & 0.378 \\
8 & 11.64 & 0.270 \\
9 & 8.17 & 0.208 \\
10 & 10.26 & 0.272 \\
11 & 10.39 & 0.242 \\
12 & 11.52 & 0.221 \\
13 & 8.92 & 0.212 \\
14 & 13.04 & 0.228 \\
15 & 10.88 & 0.251 \\
\hline
\end{tabular}

cost statistical PMD/PDL emulator was presented in Perlicki (2009). This emulator was constructed by concatenating 15 highly birefringence optical fiber segments. In Perlicki (2009) was shown that the designed emulator can mimic the polarization effects (PMD and PDL) of a real modern optical fiber link. Here, impact of combination of PMD and PDL effects on PDM transmission quality is investigated. The designed PMD/PDL emulator (Perlicki 2009) is applied to emulating of statistical nature of PMD and PDL in fiber optics links. In this paper I suggest that the proper PDM system testing should be based on simultaneous PMD and PDL effects generation. These effects should be generated by means of one device that simulates the statistical nature of polarization effects of a real optical fiber link.

\section{Statistical PMD and PDL effects emulator}

Statistical PMD and PDL effects emulator was presented in Perlicki (2009). The emulator was constructed by concatenating 15 highly birefringence optical fiber segments and randomly varying the mode coupling between them, by rotating the polarization state between them. Table 1 shows the DGD and PDL values for each birefringence segment. Figures 1 and 2 show the theoretical histograms of DGD and PDL, respectively. The theoretical frequency autocorrelation function for PMD and PDL vectors are shown in Figs. 3 and 4 respectively. This emulator was designed to have a mean DGD value of $40 \%$ of a bit time; the mean DGD value is equal to $40 \mathrm{ps}$ (Hauer et al. 2004) because the emulator was designed for testing of $10 \mathrm{Gbit} / \mathrm{s}$ Non Return Zero (NRZ) code system.

\section{Impact of polarization effects on polarization division multiplexing transmission}

\subsection{Numerical simulation results}

The impact of combination of PMD and PDL effects on PDM system quality was observed through numerical simulations of PDM transmission via the designed statistical PMD and 


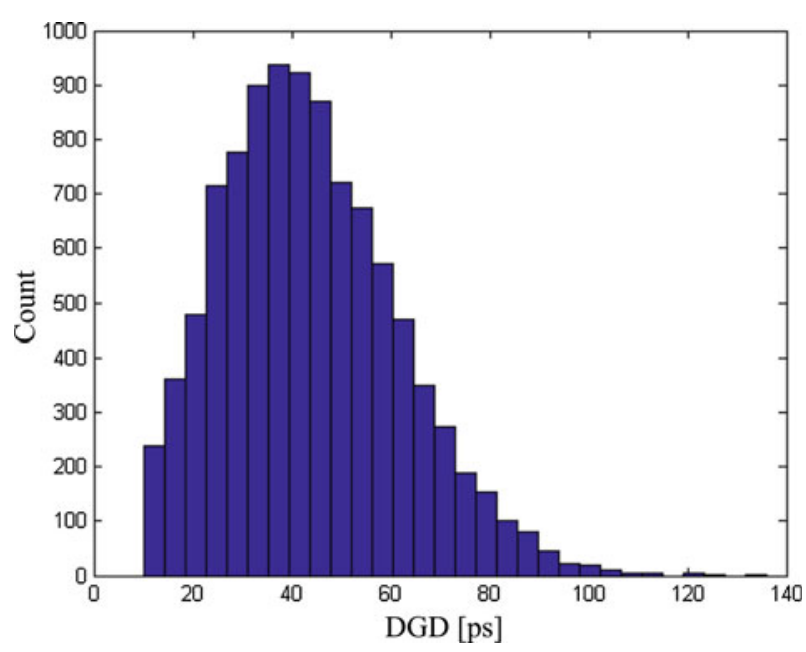

Fig. 1 Theoretical statistical distribution of DGD

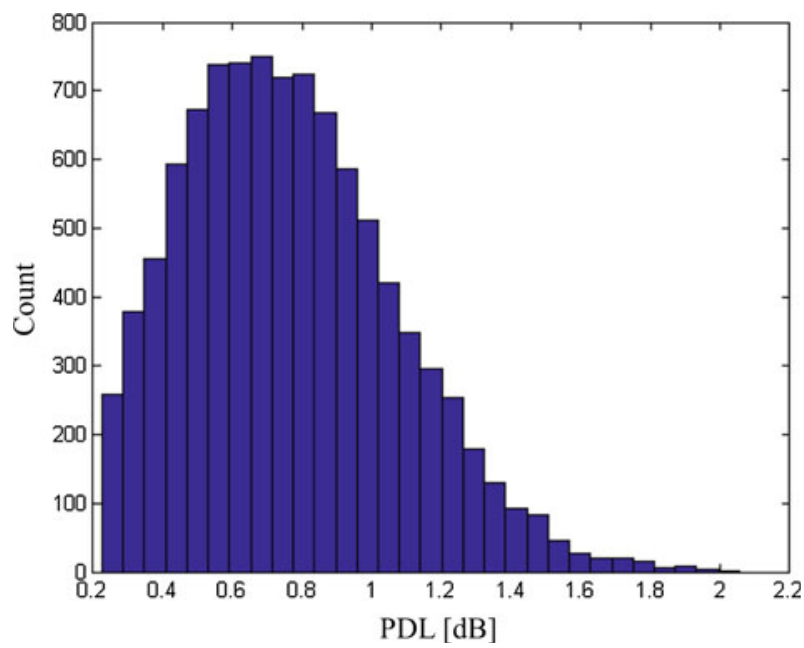

Fig. 2 Theoretical statistical distribution of PDL

PDL effects emulator. The PMD and PDL influence was simulated by means of random coupling between birefringence segments. The optical pulse propagation through our emulator was calculated by means of the following expression:

$$
\left(\begin{array}{l}
A_{x}\left(\omega, z_{n+1}\right) \\
A_{y}\left(\omega, z_{n+1}\right)
\end{array}\right)=M_{n, W P}(\omega) \cdot\left(\begin{array}{l}
A_{x}\left(\omega, z_{n}\right) \\
A_{y}\left(\omega, z_{n}\right)
\end{array}\right) .
$$

where: $M_{n, W P}(\omega)$ is the transfer matrix of $n$-th birefringence segment, $A_{x}\left(\omega, z_{n}\right)$ and $A_{y}\left(\omega, z_{n}\right)$ are the amplitudes for the two polarization components (polarization channels) at the input of $n$-th birefringence segment; in turn $A_{x}\left(\omega, z_{n+1}\right)$ and $A_{y}\left(\omega, z_{n+1}\right)$ are the amplitudes for the two polarization components at the output of $n$-th birefringence segment. 


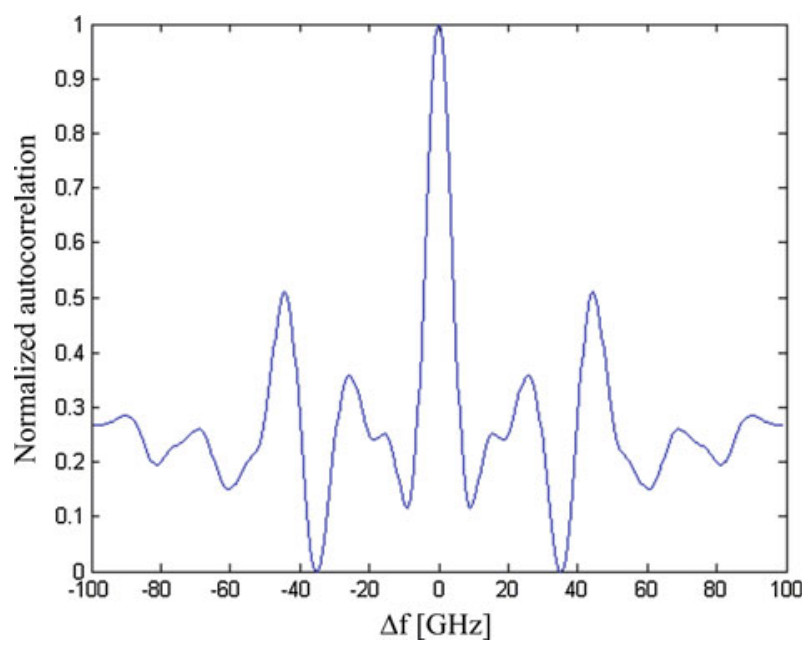

Fig. 3 Theoretical normalized frequency autocorrelation function for PMD

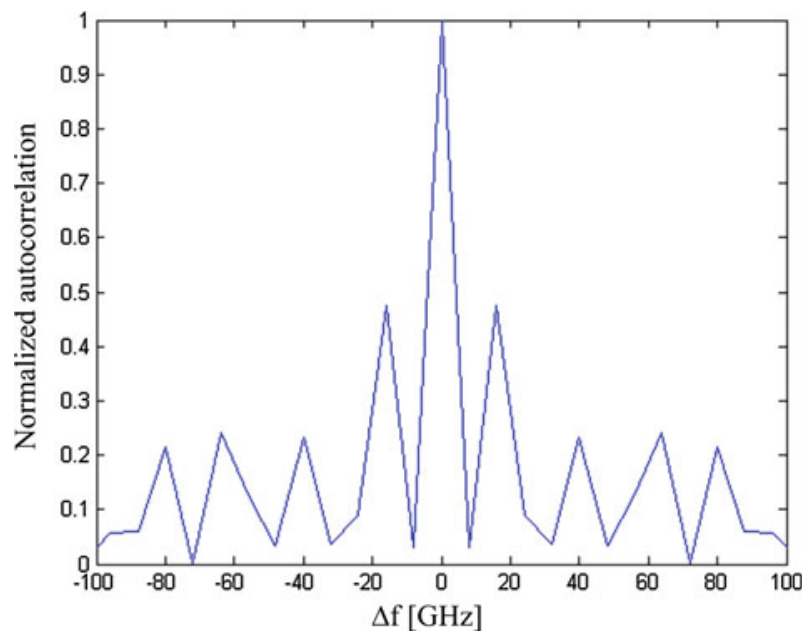

Fig. 4 Theoretical normalized frequency autocorrelation function for PDL

The transfer matrix of a single birefringence segment $\left(\mathrm{M}_{\mathrm{WP}}\right)$ can be written as:

$$
\operatorname{M}_{\mathrm{WP}}(\omega)=\mathrm{M}_{\mathrm{R}}(-\theta) \cdot \mathrm{M}_{\mathrm{PDL}} \cdot \mathrm{M}_{\mathrm{PMD}} \cdot \mathrm{M}_{\mathrm{R}}(\theta)
$$

The rotation matrix $\mathbf{M}_{\mathbf{R}(\theta)}$ is given by Collet (2003):

$$
M_{R(\Theta)}=\left[\begin{array}{ll}
\cos (\Theta) & \sin (\Theta) \\
-\sin (\Theta) & \cos (\Theta)
\end{array}\right]
$$

where $\Theta$ is the angle between the so called "fast axis" and x-axis of the reference frame. The retarder matrix $\mathrm{M}_{\mathrm{PMD}}$ is given by Collet (2003):

$$
\mathrm{M}_{\mathrm{PMD}}=\left[\begin{array}{cc}
\exp (j \tau \omega / 2) & 0 \\
0 & \exp (-j \tau \omega / 2)
\end{array}\right],
$$



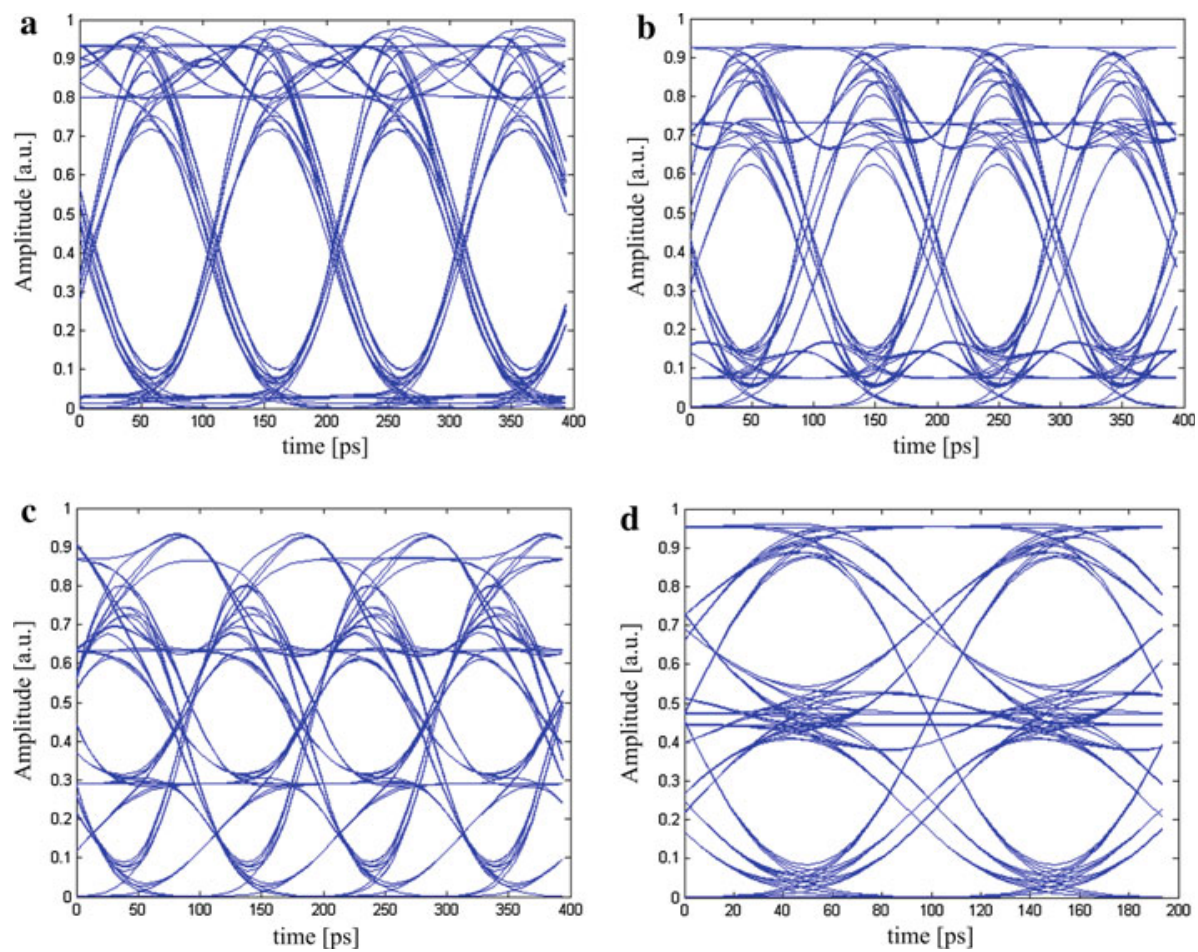

Fig. 5 Eye diagrams of demultiplexed PDM signals for different random settings of PMD/PDL emulator (numerical results)

where $\omega$ is the angular frequency and $\tau$ is the DGD. In turn, the PDL element matrix MPDL is equal to Collet (2003):

$$
\mathrm{M}_{\mathrm{PDL}}=\left[\begin{array}{cc}
\exp (-\alpha / 2) & 0 \\
0 & \exp (\alpha / 2)
\end{array}\right]
$$

where $\alpha$ is defined as: PDL $[\mathrm{dB}]=10 \log (\exp (2 \alpha))$. Here the global attenuation is neglected.

Both polarization channels consisted of a $10 \mathrm{Gbit} / \mathrm{s}$ NRZ data streams with $2^{9}-1$ Pseudo Random Bit Sequence (PRBS) length. Wavelength was $1550.0 \mathrm{~nm}$. At the transmitting side the optical channels are aligned to antipodal, orthogonal linear polarization states (linear vertical and horizontal). At the receiving end, after transmission through the designed emulator, the polarization channels were demultiplexed using polarization beam splitter. This polarization element was simulated by means of linear vertical and horizontal polarizer. Before demultiplexing, the output optical power of both polarization channels was set to be identical and maximum by means of polarization controller. The polarization controller changed the state of polarization of the output optical signal. The polarization controller was simulated by cascade of rotated quarter-, half- and quarter- waveplate. Figure 5 shows the eye diagrams for signal transmitted through the designed emulator at the receiving end after demultiplexing. We assumed the Gaussian-shaped filter at the receiver. The Gaussian shaped filter transfer function is given by: 


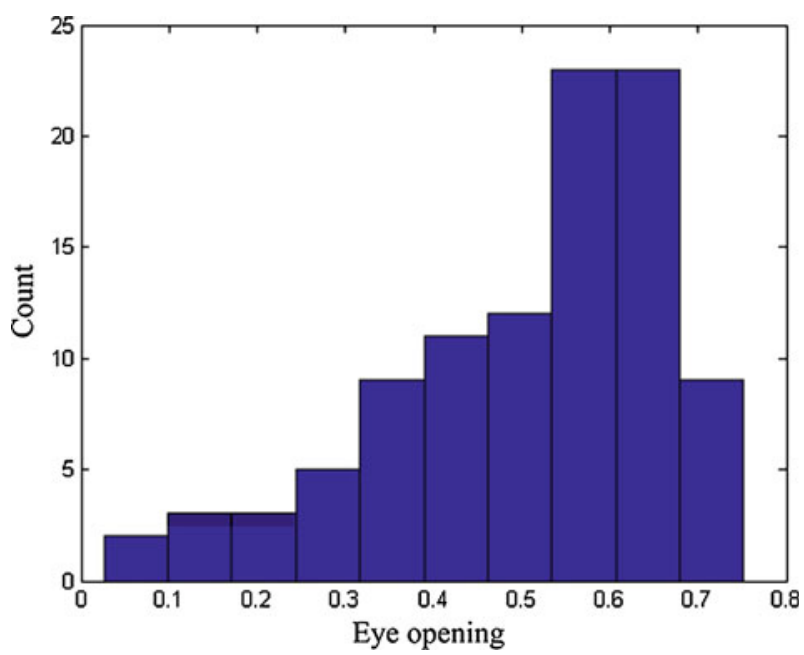

Fig. 6 Statistical distribution of simulated eye opening values for 10 Gbit/s PDM transmission

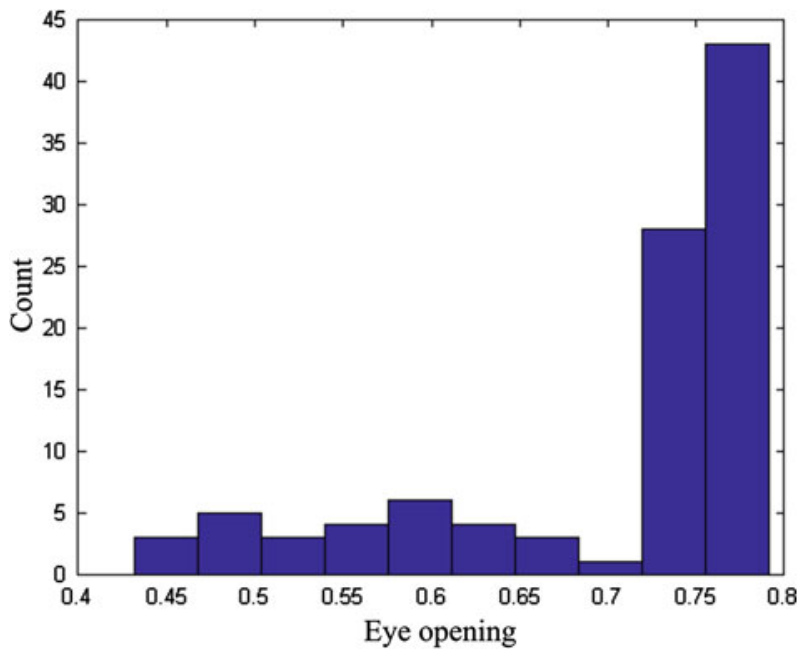

Fig. 7 Statistical distribution of simulated eye opening values for $10 \mathrm{Gbit} / \mathrm{s}$ non-PDM transmission

$$
H(\omega)=\exp \left(-\frac{\omega^{2}}{2 \omega_{\mathrm{g}}^{2}}\right),
$$

where $\omega$ is angular frequency, $\omega_{\mathrm{g}}$ is filter bandwidth. We assumed $\omega_{\mathrm{g}}=2 \pi 10 \mathrm{GHz}$.

The presented eye diagrams were created for four different random settings of our emulator.

Figure 6 shows the eye opening values statistical distribution for $10 \mathrm{Gbit} / \mathrm{s}$ PDM transmission via our PMD/PDL emulator. For comparison, Fig. 7 presents the eye opening values statistical distribution for $10 \mathrm{Gbit} / \mathrm{s}$ non-PDM single channel transmission. The eye opening values were calculated for 100 different random settings of our emulator. 


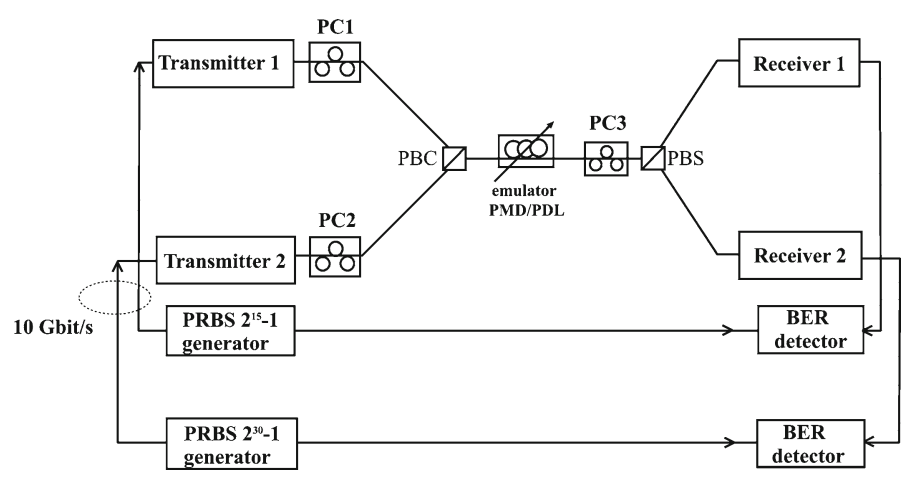

Fig. 8 Experimental setup for PDM transmission quality measurements; $P C 1, P C 2, P C 3$ Polarization Controllers, $P B C$ Polarization Beam Combiner, $P B S$ Polarization Beam Splitter, BER Bite Error Rate, PRBS Pseudo Random Bit Sequence

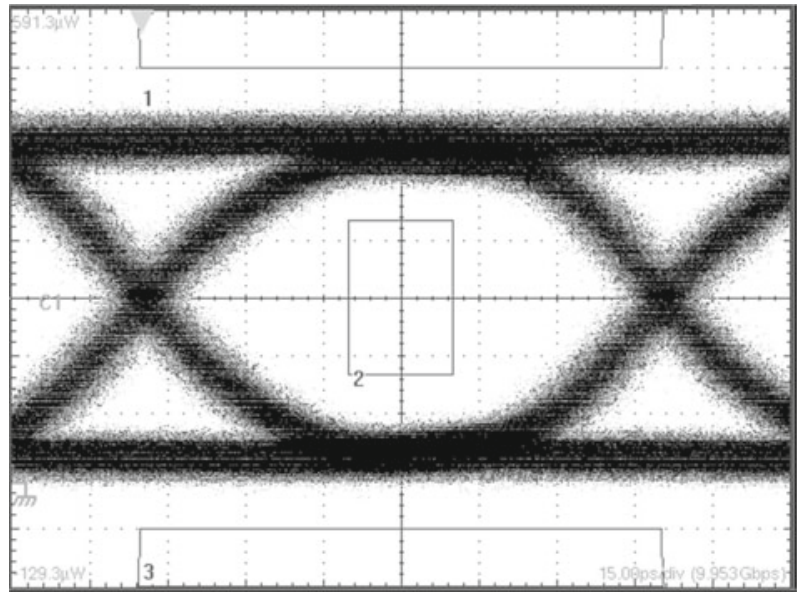

Fig. 9 Eye diagram of demultiplexed signal for back-to-back transmission

\subsection{Experimental results}

In order to evaluate the effects of PMD and PDL on an optical transmission based on polarization multiplexing technique an optical link was mounted as in Fig. 8. The optical signals from the transmitters were polarized by polarization controllers (PC1 and PC2) and multiplexed, combined onto our PMD/PDL emulator by Polarization Beam Combiner (PBC). The optical channels were aligned to antipodal, orthogonal linear polarization states (linear vertical and horizontal). At the receiving end the optical signals were demultiplexed by means of Polarization Beam Splitter (PBS). To optimize the transmission of polarization channels, the output optical power of both channels was set to be identical and maximum by adjusting polarization controller before PBS (PC3). The polarization controller PC3 was adjusted for maximum a proper polarization channel optical power and minimum crosstalk. The polarization channels were transmitted at $1550.0 \mathrm{~nm}$. In described experiment $10 \mathrm{Gbit} / \mathrm{s}$ bit rate for each polarization channel was used. The first polarization channel carried $2^{15}-1$ PRBS data stream, the second channel carried $2^{30}-1$ PRBS data stream. The eye diagram for the back to back transmission is shown in Fig. 9. In turn, Fig. 10 presents eye diagrams measured 
a

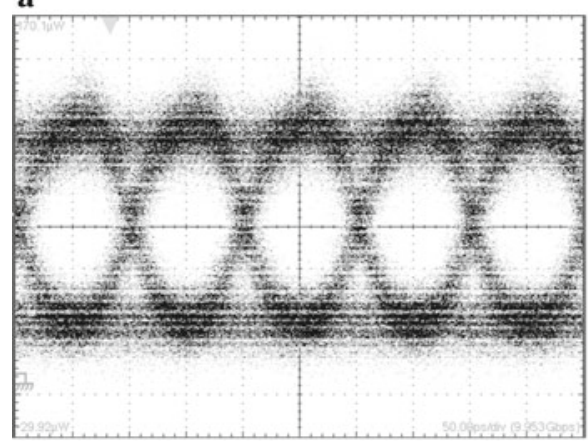

c

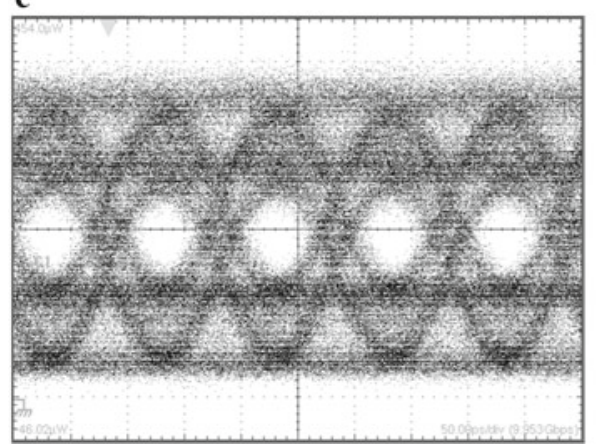

b

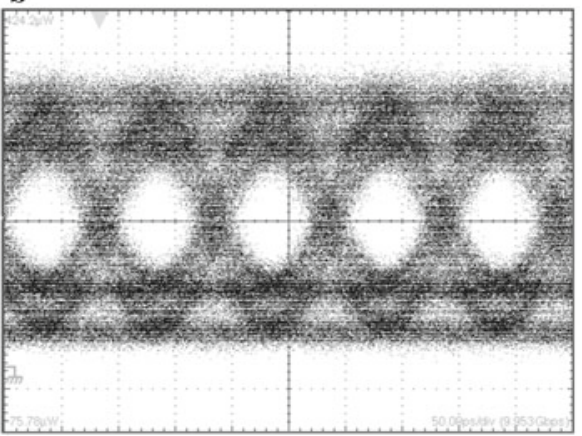

d

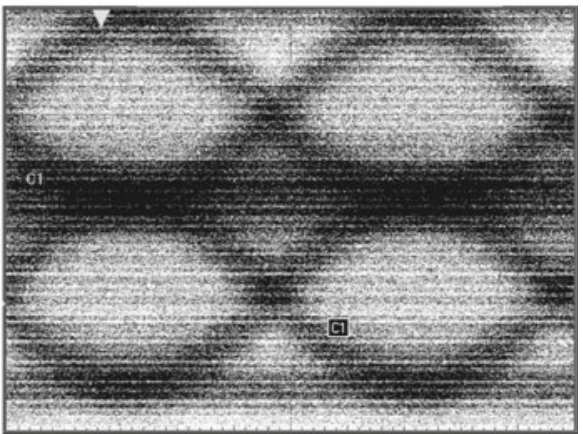

Fig. 10 Eye diagrams of demultiplexed PDM signals for different random settings of PMD/PDL emulator (experimental results); $\mathbf{d}$ eye diagram was measured for very low optical signal

for four different random settings of our emulator. The obtained eye diagrams are similar to numerical results. In Fig. 10a, b and c we can see the different impact of a pure PMD on bit transmission. In turn in Fig. 10d dominates crosstalk between polarization channels. The impact of polarization effects on PDM system quality was assessed by means of bit error rate (BER) measurement. We realize that the birefringence in optical fibers (in our emulator) induces an upredictable rotation to state of polarization with time. This rotation should be continuously corrected in order to separate both polarization channels. Additionally, here no feedback loop was available on the PC3 preceding demultiplexing PBS. For this reason the BER measurements were performed over relatively short time periods i.e. 100 s. Figure 11 shows the BER statistical distribution for $10 \mathrm{Gbit} / \mathrm{s}$ PDM transmission via our PMD/PDL emulator. The BER values were measured for 100 different random settings of our emulator. The results suggest that PDM systems are very sensitive to both PMD and PDL effects. For comparison, Fig. 12 presents the BER statistical distribution for 10 Gbit/s non-PDM single channel transmission. The presented BER values were also measured for 100 different random settings of our emulator. We can see that $10 \mathrm{Gbit} / \mathrm{s}$ PDM system is more sensitive to polarization effects than $10 \mathrm{Gbit} / \mathrm{s}$ single channel system. Our results and analysis which was presented in Nelson et al. (2001) suggest that PDM systems are a factor of five more sensitive to PMD that non-PDM single channel transmission systems.

There are several polarization mechanisms leading to impairments in PDM systems (van den Borne et al. 2005, 2004; Tuft et al. 2006; Nelson et al. 2000): 


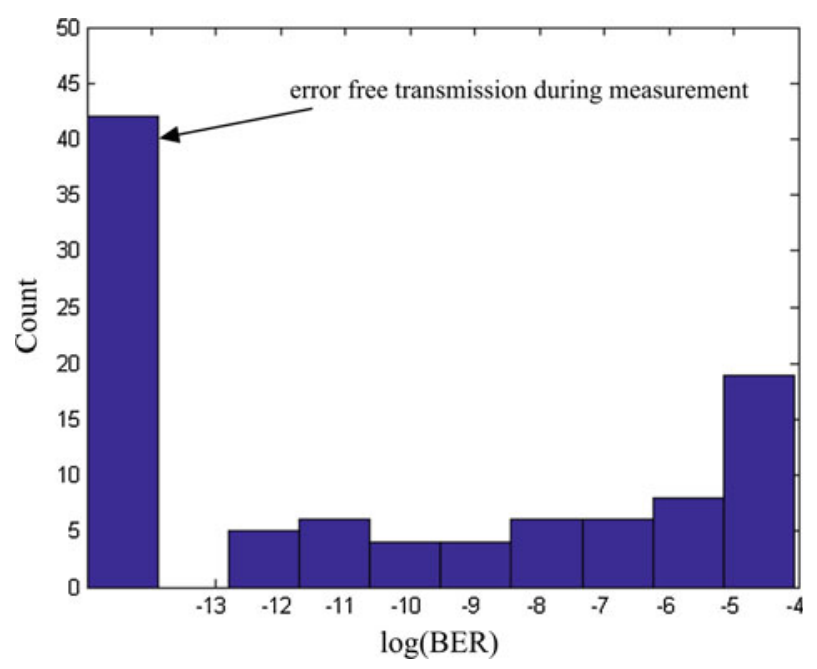

Fig. 11 BER statistical distribution for $10 \mathrm{Gbit} / \mathrm{s}$ PDM transmission

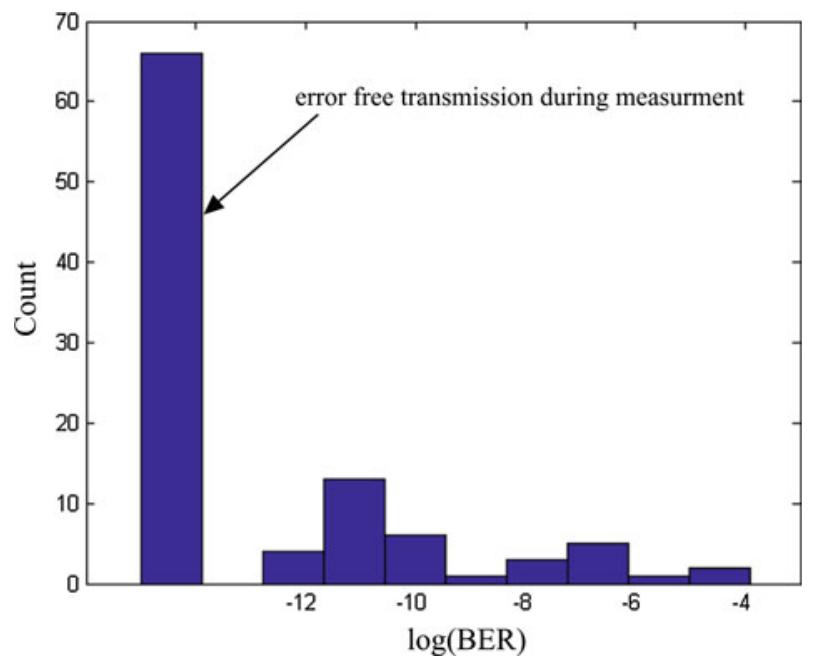

Fig. 12 BER statistical distribution for $10 \mathrm{Gbit} / \mathrm{s}$ non-PDM single channel transmission

(1) The PMD and PDL effects cause inter symbol interference, similar to single channel transmission. Combined PMD/PDL effect degrade the optical system more than either PMD or PDL alone.

(2) Due to PMD, the pulses partly overlap and the State of Polarization (SOP) of the overlapping part changes with respect to the SOP of the center part of the pulses. The output polarization controller cannot change the SOP on the bit time scale. The alignment with the PBS is not optimal near the edge of the pulse, which results in suboptimal demultiplexing and the introduction of coherent crosstalk. This is so called edge effect.

(3) The PDL effect impairs the orthogonality between multiplexed polarization channels.

(4) The optical fiber birefringence induces the SOP changing with time; an upredictable SOP rotation leading to PBS misalignment. 
(5) The SOP for carrier is aligned with the axes of the PBS but the PMD effect induces a frequency dependence of SOP. In this situation an optical signal is coupled from polarization channel to the other as frequency deviate from the carrier.

\section{Conclusion}

Impact of the PMD and PDL effects on $10 \mathrm{Gbit} / \mathrm{s}$ PDM and non-PDM single channel transmission system was evaluated by means of the designed PMD/PDL emulator. The results show that PDM system transmission is very sensitive to both PMD and PDL effects. Furthermore, the achieved results suggest that PDM system transmission is more sensitive to polarization effects that non-PDM single channel system transmission. We can find information about PDM system testing by means of PMD emulators and/or PDL emulators. But this is not correct. The proper PDM system testing should be based on simultaneous PMD and PDL effects generation. These effects should be generated by means of one device that simulates the statistical nature of polarization effects of a real optical fiber link.

Open Access This article is distributed under the terms of the Creative Commons Attribution Noncommercial License which permits any noncommercial use, distribution, and reproduction in any medium, provided the original author(s) and source are credited.

\section{References}

Collet, E.: Polarized Light in Fiber Optics. The PolaWave Group, Lincroft, NJ (2003)

Hauer, M.C., et al.: Electrically controllable All-Fiber PMD Emulator using a compact array of thin-film microheaters. J. Lightw. Technol. 4, 1059-1065 (2004)

Lima, I.T.: Comparison of polarization mode dispersion emulators. J. Lightw. Technol. 12, 1872-1881 (2001)

Nelson, L.E., et al.: Coherent crosstalk impairments in polarization multiplexed transmission due to polarization mode dispersion. Opt. Express 10, 350-361 (2000)

Nelson, L.E., et al.: Observation of PMD-Induced Coherent Crosstalk in Polarization-Multiplexed Transmission. IEEE Photon. Technol. Lett. 7, 738-740 (2001)

Palmer, L., et al.: Frequency-correlation analysis of PMD emulators with symmetric polarization scrambling. J. Lightw. Technol. 11, 3897-3906 (2006)

Perlicki, K.: Statistical PMD and PDL effects emulator based on polarization maintaining optical fiber segments.. Opt. Quant. Electron. 1, 1-10 (2009)

Tuft, V.L., et al.: The effect of PDL in a polarization and time division multiplexed scheme for all-optical class of service segregation. In: International Conference of Transparent Optical Network, pp. 91-96 (2006)

van den Borne, D., et al.: PMD-Induced transmission penalties in polarization-multiplexed transmission. J. Lightw. Technol. 12, 4004-4014 (2005)

van den Borne, D., et al.: PMD and nonlinearity-induced penalties on polarization-multiplexed transmission. IEEE Photon. Technol. Lett. 9, 2174-2176 (2004) 